\title{
Proposta de atividade para o quinto ano do ensino fundamental: Algoritmos Desplugados ${ }^{*}$
}

\author{
Gustavo Santos ${ }^{2}$, Wine Silva ${ }^{2}$, Simone Cavalheiro ${ }^{1,2}$, \\ Luciana Foss $^{1,2}$, Marilton Aguiar ${ }^{1,2}$, Ana Marilza Pernas ${ }^{1,2}$, \\ André Du Bois ${ }^{1,2}$, Renata Reiser ${ }^{1,2}$ \\ ${ }^{1}$ Programa de Pós Graduação em Computação \\ ${ }^{2}$ Centro de Desenvolvimento Tecnológico - Universidade Federal de Pelotas (UFPEL) \\ Rua Gomes Carneiro, 1 - 96.010-610 - Pelotas - RS - Brasil \\ \{gfdsantos, wdssilva, simone.costa\}@inf.ufpel.edu.br
}

\begin{abstract}
This paper presents an activity to be developed in public schools, for the fifth year of elementary school, aiming to develop Computational Thinking skills teaching Algorithms. The study is part of a university extension project that proposes to promote the methodology of Computational Thinking. Our methodology uses the computer science unplugged skills, in which is taught computing concepts without the use of computers, considering fun tasks. As educational material, we have built blocks to define algorithms, similar to those found in software like Scratch.
\end{abstract}

Resumo. Este artigo apresenta uma proposta de atividade a ser desenvolvida em escolas públicas, para turmas do quinto ano do Ensino Fundamental, com objetivo de promover habilidades do Pensamento Computacional através do ensino de Algortimos. O trabalho faz parte de um projeto de extensão universitária no qual propõe-se promover a metodologia do Pensamento Computacional. Como metodologia, foi utilizado o conceito de Computação Desplugada, no qual é ensinado conceitos de computação sem o uso do computador, com tarefas lúdicas e divertidas. Como material didático foram desenvolvidos blocos para a construção de algoritmos, semelhantes aos encontrados em softwares como Scratch.

\section{Introdução}

A crescente presença e influência de ferramentas computacionais em atividades cotidianas vem alterando o perfil de inúmeras áreas. Diversas escolas, por exemplo, já possibilitam aos alunos o contato com computadores e softwares. No entanto, se limitam a este tratamento [Costa et al. 2012]. Desta forma, defende-se a necessidade de apresentar a Computação, não apenas como um instrumento, mas também como uma forma de solucionar, de forma eficiente, problemas [Linn et al. 2010]. Sob esse panorama, o Pensamento Computacional surge como um proposta para difusão dessa nova abordagem.

O Pensamento Computacional pode ser entendido como uma habilidade primordial a ser dominada por todos, tão necessária quanto habilidades aritméticas básicas ou a

\footnotetext{
*Projeto realizado com o apoio do PROEXT - MEC/SESu, PICMEL - FAPERGS/CAPES e da Universidade Federal de Pelotas
} 
capacidade de ler e escrever [Wing 2006]. Visando incorporar o Pensamento Computacional no currículo escolar desde as séries iniciais, associações como Computer Science Teachers Association e International Society for Tecnology in Education com suporte da National Science Foundation criaram o Computational Thinking in K-12 Education - Leadership Toolkit. Este Toolkit [CSTA et al. 2010] define uma série de habilidades essenciais para a introdução e disseminação do Pensamento Computacional no contexto escolar. Para trabalhar habilidades do Pensamento Computacional sem utilizar computadores, foi desenvolvido a técnica de Computação Desplugada [Bell et al. 2011]. Utilizando como base a técnica de Computação Desplugada, esta atividade foi desenvolvida com o objetivo de ensinar os conceitos das áreas da Ciência da Computação sem a necessidade do uso de computadores, evidenciando o caráter científico da mesma.

Projetos que integrem o Pensamento Computacional como um conceito no Ensino Fundamental são de suma importância, uma vez que as habilidades trabalhadas no Pensamento Computacional são equivalentes a habilidades básicas [Wing 2006]. Segundo Bezerra, é importante a discussão sobre a introdução do Pensamento Computacional como disciplina obrigatória para o ensino fundamental [Bezerra 2014]. O restante deste trabalho aborda uma proposta de atividade que desenvolve o Pensamento Computacional, visando turmas do quinto ano do Ensino Fundamental de escolas públicas.

\section{Pensamento Computacional}

Pensamento Computacional foi definido como um conjunto de técnicas que utiliza fundamentos da Ciência da Computação para resolução de problemas gerais [Wing 2006]. Segundo Wing, o Pensamento Computacional é uma habilidade básica fundamental [Wing 2006], seguindo esta linha de raciocínio, então é possível relacionar este diretamente ao ensino na Educação Básica, como forma de tornar o exercício do Pensamento Computacional ativo e visível no cotidiano brasileiro.

O trabalho do Pensamento Computacional na Educação Básica vem ao encontro das habilidades primariamente trabalhadas em sala de aula, uma vez que as habilidades básicas do Pensamento Computacional são trabalhadas no cotidiano escolar [SEF 1997], apesar deste não se caracterizar uma norma para a toda a Nação [SEB et al. 2013], muitos sistemas de ensino baseiam seus currículos nesses parâmetros.

Como habilidades necessárias a profissionais da área de computação, pode-se elencar as seguintes técnicas pertencentes ao Pensamento Computacional [CSTA et al. 2010]: coleta de dados, análise de dados, representação de dados, decomposição de problemas, abstração, algoritmos, simulação e paralelização.

\section{Motivação e Trabalhos Relacionados}

A motivação para o desenvolvimento desta proposta de atividade é o avanço na comunidade científica sobre a computação na educação de crianças e adolescentes. Muitos dos trabalhos revisados mais recentes reportam avanços importantes, como o ensino de conceitos da Computação a professores para que os mesmos o apliquem em sala de aula, o ensino da programação na pratica usando o Scratch ou mesmo programando um chip.

Silva et al. [Silva et al. 2014b] relata uma experiência de realização de oficinas que deram a oportunidade a professores da rede estadual e municipal de ensino do Rio 
Grande do Norte a utilizar o software Scratch, como ferramenta auxiliar no ensino. Assim como neste trabalho, os autores utilizaram tarefas lúdicas para o ensino de computação utilizando a Computação Desplugada, onde são ensinados conceitos de computação, sem o uso de computador. O trabalho também mostra resultados significativos no interesse dos professores nos conceitos transmitidos ao longo das oficinas, bem como a proposta aos autores para a realização das oficinas juntamente dos alunos e a junção do ensino de conceitos de Computação e outras disciplinas presentes no currículo dos estudantes [Silva et al. 2014b].

O trabalho de Scaico et al. propõe o ensino de programação no ensino médio através de técnicas básicas de programação, como o uso de variáveis, funções, entrada e saída de dados, estruturas de repetição e controle para desenvolver habilidades de programação [Scaico et al. 2012], para desenvolver habilidades de decomposição, abstração e construção de algoritmos, pertencentes ao Pensamento Computacional. No trabalho, os autores relatam a ocorrência de uma olimpíada de programação, como motivação aos participantes do curso a demonstrar seus conhecimentos adquiridos.

Sousa [Sousa 2013] em seu trabalho mostrou suas experiências na realização de um curso de introdução a algoritmos, onde foi oferecido a alunos do ensino médio de uma escola pública, desenvolvendo técnicas de lógica e algoritmos, que são habilidades do Pensamento Computacional. Sousa focou em exercícios de lógica e algoritmos básicos e a avaliação dos conhecimentos adquiridos pelos alunos foi satisfatória, motivando os autores a prosseguir com o projeto [Sousa 2013].

Torezani et al. em seu trabalho apresenta o desenvolvimento do software para auxilio pedagógico: NewProg [Torezani et al. 2013]. Segundo Torezani et al., o uso do NewProg favoreceu o desenvolvimento de habilidades cognitivas, em crianças com faixa etária de cinco a oito anos de idade, que são necessárias à resolução de problemas.

Já Viel descreve em seu trabalho a iniciação de estudantes do ensino médio em conceitos de programação de computadores, bem como o desenvolvimento de projetos de circuitos digitais e implementação de um processador com fins educativos [Viel et al. 2014]. O projeto utilizou o processador BIP para o auxílio pedagógico no desenvolvimento do pensamento computacional. $\mathrm{O}$ trabalho proposto por Viel [Viel et al. 2014], além de introduzir conceitos de programação, propõe o desenvolvimento do pensamento computacional através do uso de uma ferramenta computacional para a programação do processador BIP. Esta proposta se mostrou adequada para pequenos grupos de estudantes, embora a quantidade não seja apontada pelo autor.

Silva et al. [Silva et al. 2014a] em seu trabalho propõe o uso do Scratch para o ensino de programação a professores do quinto ano do ensino fundamental. O objetivo do trabalho foi proporcionar aos professores participantes, de distintas áreas do conhecimento, como pedagogia, geografia, física, história, matemática e português, a utilização do Scratch. Em seu projeto, [Silva et al. 2014a] mostra que os cinco professores, da escola onde o projeto foi aplicado, apontaram que os objetivos apresentados para a aprendizagem são considerados importantes, além de apontar sugestões para o aprimoramento do trabalho, como incorporação ao ensino de matemática e física, mais precisamente na aritmética e cinemática. Dentre os objetivos considerados importantes pelos professores participantes, estão: possibilidade do aluno criar seu próprio jogo, a utilização da ferra- 
menta na aprendizagem dos alunos, a possibilidade de trabalhar conteúdos de aula através de jogos, dentre outros. Embora a proposta da atividade apresentada neste trabalho não exponha aplicações diretas em disciplinas curriculares dos alunos do quinto ano do ensino fundamental, diversos exemplos didáticos e lúdicos serão considerados durante as aulas para exemplificar a aplicação de algoritmos no cotidiano escolar de cada aluno.

Outros trabalhos de [Andrade et al. 2013] e [Gonçalves et al. 2013] também focaram na promoção de habilidades do Pensamento Computacional, propondo e aplicando atividades fundamentadas em conceitos da computação.

Como visto acima, trabalhos diferentes apresentam aproximadamente a mesma característica, o ensino de programação utilizando alguma ferramenta computacional. Diferente da abordagem apontada pelos autores citados, esta proposta visa o ensino de algoritmos e programação sem fazer uso de qualquer ferramenta computacional.

\section{Metodologia}

Para a construção da atividade Algoritmos Desplugados, foi feito um levantamento de fundamentações teóricas necessárias a se incluir nesta atividade, principalmente sobre o Pensamento Computacional.

Com a necessidade de incluir o Pensamento Computacional em escolas, esta proposta foi desenvolvida visando o quinto ano do Ensino Fundamental de escolas públicas. Entretanto, não são todas as escolas públicas que contém uma infraestrutura adequada para a realização de atividades utilizando o computador, pensando nisso é proposto neste trabalho uma atividade que trabalha questões do Pensamento Computacional, referentes a algoritmos, sem fazer o uso do computador. Para isto foi feito um levantamento das condições de escolas públicas da rede municipal de Pelotas para adequar todas as tarefas contidas nesta atividade ao cotidiano dos alunos.

Utilizou-se como inspiração para esta atividade o software Scratch, mais precisamente o modo de construção de algoritmos, utilizando blocos. Na atividade Algoritmos Desplugados, os comandos são representados por blocos de EVA de diferentes cores e tamanhos, onde cada bloco é encaixado em outro e um conjunto de blocos devidamente encaixados, forma um algoritmo. Para a execução dos algoritmos é utilizado diversos tabuleiros impressos em folhas de tamanho A4, além de itens coletáveis e desafios. O centro da atividade é o personagem Jack, onde este é representado por uma figurinha disposta no mapa capaz de executar os algoritmos desenvolvidos. A atividade Algoritmos Desplugados dispões de uma série de tarefas responsáveis por ensinar as estruturas básicas: repetição e decisão, através de algoritmos imperativos.

\section{Proposta}

A proposta da atividade Algoritmos Desplugados foi desenvolvida levando em conta a infraestrutura das instituições de ensino público pelotense e o perfil de interesse dos alunos participantes pelo material utilizado em atividades anteriores realizadas pelo grupo EXP-PC [EXP-PC 2015].

A divisão da proposta foi efetuada em sete tarefas, onde há uma avaliação sobre os conceitos transmitidos e uma tarefa que visa revisar os conceitos transmitidos ao longo da atividade. As demais tarefas são divididas de acordo com tópicos, que são: 
variáveis, sequenciamento, repetições e decisões, abordando os seguintes itens do Pensamento Computacional: decomposição, abstração, algoritmos e padrões [Wing 2011]. As tarefas propostas são descritas a seguir.

\subsection{Origamis como Algoritmos}

Objetivo. A primeira tarefa da atividade Algoritmos Desplugados propõe o uso de origamis como introdução ao sequenciamento de passos. No total são utilizados seis modelos de origamis diferentes, cada um com um algoritmo próprio e ilustrado, como um exemplo exibido na Figura 1.

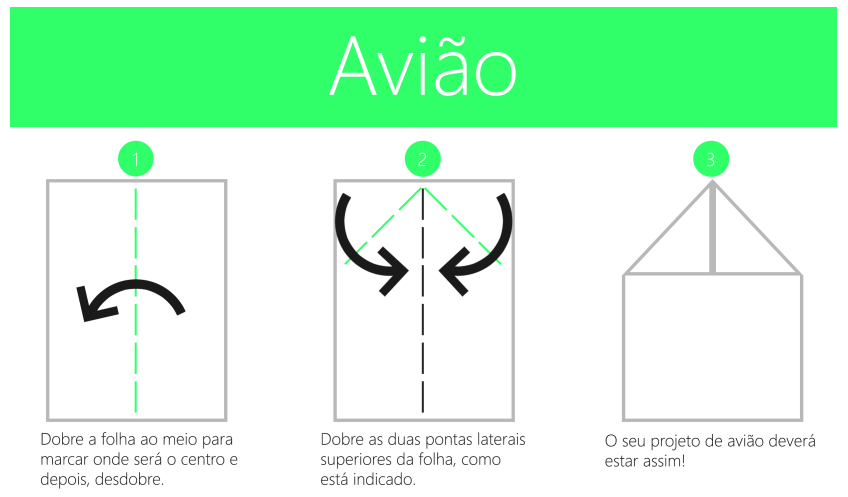

Figura 1. Trecho do origami do Avião

Os demais modelos de origamis estão disponíveis do sitio do projeto EXP-PC [EXP-PC 2015].

Metodologia. A primeira tarefa introduz algoritmos através da construção de origamis. Inicialmente os alunos são questionados sobre ações do cotidiano que evidenciem a sequência de passos que todos os dias executam para um determinado fim, por exemplo, "qual a sequência de passos para fazer um bolo?", "qual a sequência de passos para ir a uma festa?" ou "qual a sequência de passos para ir a escola?". Depois de estabelecida essa sequência de passos, o professor deve esclarecer que todo o dia executamos algum algoritmo para obter algum resultado e que, muitas vezes, quando trocamos a ordem de execução de algum passo do algoritmo, podemos obter um resultado longe do desejado. Exemplificando com o algoritmo de fazer um bolo: "se invertemos a ordem de algum passo no algoritmo de fazer um bolo, o que pode acontecer com o nosso resultado?". O ministrante da aula deve, então, questionar se os alunos conhecem origamis, ou se já realizaram dobraduras de papel, como por exemplo, um avião de papel ou um barco de papel. Feitas estas considerações, apresenta-se aos alunos os algoritmos dos origamis a serem trabalhados; os origamis servem para corroborar a ideia de que quando a sequência está bem definida, todos chegam ao mesmo resultado sempre que executarem o algoritmo. São trazidos pelo professor vários algoritmos para a elaboração de origamis, estes algoritmos vêm ao encontro da explicação de algoritmos e como eles precisam ser bem definidos e claros para que todos cheguem ao mesmo resultado, os questionamentos enfoque desta aula devem ser "Como formular uma sequência bem defina de passos?", "Por que precisamos definir claramente as instruções que damos a outras pessoas?" ou então "Por que formar sequências bem definidas de passos?". 

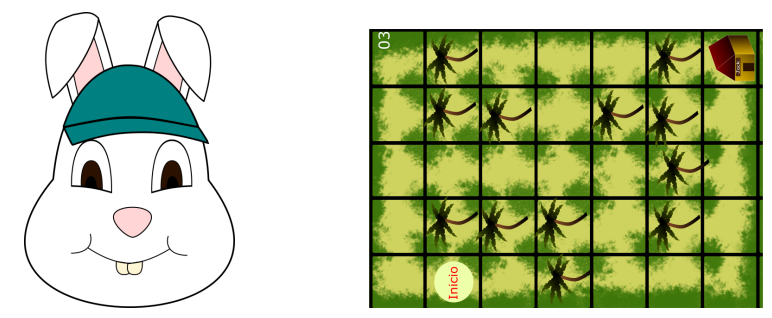

Figura 2. Personagem Jack e um modelo de tabuleiro.

Materiais. Para esta tarefa são necessárias folhas com o passo-a-passo de cada origami, sugerindo-se que seja de uma gramatura mais elevada e plastificada para uma reutilização posterior; também são necessárias folhas, preferencialmente A4 de cores diferentes para a elaboração dos origamis.Na falta de folhas A4 em cores diversas, pode ser utilizado, lápis de cor para os alunos pintarem os origamis.

\subsection{Jack, O Coelho}

Objetivo. Nesta tarefa é apresentado o personagem Jack, que servirá de motivação para todas as próximas tarefas desta atividade. O objetivo desta tarefa consiste em os alunos proporem algoritmos que solucione problemas para este personagem. Em um primeiro momento, os problemas serão de resolução simples como "Faça Jack chegar na sua casa", utilizando de algoritmos puramente sequenciais, sem estruturas de repetições, decisões ou uso de variáveis, pois estas ferramentas serão introduzidas em tarefas ao longo da atividade Algoritmos Desplugados. Também são apresentas missões que exigem o desenvolvimento de algoritmos sequenciais para sua solução. Nesta tarefa são apresentadas as peças para elaboração dos algoritmos, estas peças em EVA contêm instruções para a montagem de algoritmos.

Metodologia. A tarefa, chamada de "Jack, o Coelho", se inicia com o professor relembrando os conceitos aprendidos na tarefa anterior, "Origamis como Algoritmos", e como a construção de origamis exemplificava o conceito de algoritmos. O ministrante da tarefa descreve o mapa de Jack, explicando cada item presente no tabuleiro, como quadros que o personagem não pode entrar, o ponto inicial de cada tabuleiro e o destino que o personagem deve alcançar ao final do algoritmo. Várias configurações de tabuleiros podem ser criadas a partir do modelo inicial do tabuleiro e é pedido que cada aluno anote os algoritmos criados por eles e o mapa para qual o algoritmo foi criado para fim de avaliação. Para a construção dos algoritmos, serão utilizadas instrução de "Avance", "Vire a direita"e "Vire a esquerda".

Materiais. Esta atividade requer a confecção de várias configurações de tabuleiro, cartas com o personagem Jack de tamanho $4 \times 4 \mathrm{~cm}$, para simulação do algoritmo e peças de instruções em EVA, que servem como os comandos "Avance", "Vire a direita"e "Vire a esquerda"para a construção dos algoritmos. O tabuleiro consiste um terreno sob uma malha quadriculada $5 \times 7 \mathrm{~cm}$, com quadrados de lado 4, e a indicação de caminhos entre todos os quadrados, esses quadrados podem conter obstáculos ou quadrados livres que podem ser percorridos pelo personagem. 


\subsection{Jack e seus itens}

Objetivo. Com a motivação do personagem Jack, será introduzido o conceito de variáveis como a quantidade de itens que o personagem carrega ao longo da execução do algoritmo. Esta tarefa trata também do local de armazenamento destes itens para seu uso posteriormente durante a execução do algoritmo. O objetivo é apresentar a necessidade de armazenar informações durante a execução de um algoritmo.

Metodologia. A tarefa Jack e Seus Itens busca a introdução do conceito de variável, para isso são adicionadas as instruções do personagem "Colete Item no Quadro a Frente" e "Use Item no Quadro a Frente", que servem como ações de uso e coleta de itens no tabuleiro, bem como uma carta de jogo, "Mochila", que servirá de local de armazenamento dos itens coletados, apresentando de maneira implícita o conceito de memória. A tarefa consiste na utilização de tabuleiros com novos itens: "Chave"e "Porta", onde servirão para estimular a coleta de itens e seu armazenamento. As Chaves devem ser coletadas com a instrução "Colete Item no Quadro a Frente" e utilizadas nas Portas com a instrução "Use Item no Quadro a Frente", estas instruções devem, respectivamente, incrementar e decrementar o contador do item e remover o elemento contido no quadro a frente.

Materiais. A tarefa "Jack e seus Itens" necessita da confecção de outros tabuleiros, que diferem da primeira tarefa apenas no acréscimo das chaves e das portas no tabuleiro; também devem ser acrescentados ao conjunto de instruções as peças em EVA "Colete Item a Frente" e "Use Item a Frente", bem como a nova carta "Mochila", de dimensões maiores que as demais cartas.

\subsection{Jack e suas decisões}

Objetivo. Esta tarefa tem como objetivo introduzir estruturas de decisão utilizando o personagem Jack, apresentado em tarefas anteriores. Estes questionamentos estão diretamente relacionados com Estruturas de Decisão, que será o tema abordado nesta tarefa.

Metodologia. A quarta tarefa busca introduzir as estruturas de condicional para a construção dos algoritmos. Assim como nas demais tarefas, Jack será o tema principal da tarefa e a introdução do conceito é feita a partir de problemas que obriguem o uso destas estruturas para a resolução. A estrutura de decisão servirá para verificar a veracidade de uma premissa, se esta for verdadeira, será executado uma sequência de instruções, caso contrário, será executado outra sequência de instruções. Com o objetivo de introduzir o condicional e estimular a utilização da estrutura na construção dos algoritmos, será introduzido o item "Pedra"ao tabuleiro, este item possui somente dois estados "Grande" ou "Pequeno" - caso o item "Pedra"for "Grande", o personagem deverá desviar, tomando outro caminho. Caso contrário, o personagem poderá seguir seu caminho. Este item "Pedra"será representado por uma carta virada de cabeça para baixo e seu valor somente será descoberto após desvirar a carta no tabuleiro, durante a execução do algoritmo. Pedese que cada aluno anote os algoritmos feitos e o número do mapa para cada algoritmo porque haverá diferentes modelos de mapas, para ser entregue ao final da tarefa.

Materiais. Esta atividade requer a produção de novos tabuleiros contendo todos os itens já inseridos nas atividades anteriores, acrescido do item PEDRA. Também é necessária a introdução de novas instruções ao conjunto de instruções em EVA, as instruções "Se Então" e "Se - Então - Senão". 


\subsection{Jack e suas repetições}

Objetivo. Jack necessita percorrer o tabuleiro atrás de itens necessários para completar tarefas descritas nos problemas e ao fim apresentar um algoritmo que resolva o problema. Isto será feiro através da inclusão das instruções "Enquanto"e "Repita $n$ Vezes", onde $n$ é o número de repetições de uma sequência de instruções. Esta tarefa apresentará as estruturas de repetição como um modo de diminuir a quantidade de peças utilizadas para construir algoritmos. Assim como nas demais atividades, vários problemas serão descritos para a resolução por parte dos alunos.

Metodologia. A última tarefa desta atividade aborda as estruturas de repetição, para isso são introduzidas ao jogo as peças de instrução "Enquanto" e "Repita $n$ Vezes". Nesta tarefa, são propostos problemas que envolvam coletar vários itens pelo mapa, possibilitando o uso de laços de repetição através das novas instruções, também são diminuídas as quantidades de peças para a construção dos algoritmos, induzindo a utilização de repetições.

Materiais. A tarefa necessitará de novas configurações de tabuleiro contendo maçãs, cenouras, chaves e portas. Também, serão necessárias todas as cartas e instruções previamente elaboradas para o jogo, além das instruções incluídas nesta tarefa.

\subsection{Revisão}

Esta tarefa consiste em uma revisão de todos os conceitos vistos em tarefas anteriores: algoritmos sequenciais, variáveis, estruturas de decisão e estruturas de repetição. Esta aula se destina a realização de alguns exercícios, afim de relembrar os alunos dos conceitos estudados em tarefas anteriores.

\section{Avaliação}

Para avaliação da atividade Algoritmos Desplugados, será aplicado um teste contendo questões com intuito de mensurar o nível de aprendizado dos alunos. Esta avaliação consistirá em questões dissertativas a respeito dos conceitos aprendidos. Haverá nesta avaliação, tabuleiros reduzidos com pequenos problemas para evitar a montagem de peças.

Para a avaliação da construção de algoritmos sequenciais, sugere-se dado um tabuleiro $2 \times 4$ quadrados, com um ponto inicial no primeiro quadrado superior esquerdo, propor o problema "Escreva um algoritmo que faça Jack chegar até sua casa". Para avaliar a aprendizagem de conceitos de variáveis, propõe-se um tabuleiro com vários itens a serem coletados, e fazer o questionamento "Se Jack coletar todos os itens do tabuleiro, qual será a quantidade de cada item na mochila quando chegar a sua casa?". A fim de avaliação de estruturas de decisão, propõe-se a apresentação de um algoritmo, construído a partir dos blocos utilizados pelos alunos durante a atividade, impresso na folha de exercícios com a estrutura de decisão com o seguinte questionamento "O que ocorre quando a resposta do teste é falsa? E o que acontece quando a resposta é verdadeira?". Para avaliação da estrutura de repetição propõe-se um tabuleiro e um algoritmo, os alunos deverão completar espaços em branco para que o algoritmo resolva o problema proposto, o algoritmo deve sugerir que os alunos completem o espaço com uma estrutura de repetição. A avaliação também fará uso de questões dissertativas, como "O que é um algoritmo?" e "O que devemos usar quando queremos repetir várias vezes um bloco de instruções?". 
A nota final de cada aluno será computada da seguinte forma: $70 \%$ será dedicada a avaliação final e o restante será distribuído de acordo com a participação de cada aluno nas tarefas realizadas durante a atividade.

\section{Considerações finais}

Conforme apresentado em [Silva et al. 2011], a metodologia tradicional de ensino de algoritmos e programação segue um determinado padrão: a definição formal de algoritmos e a resolução de problemas em uma linguagem de programação. A atividade Algoritmos Desplugados pretende ensinar a definição de algoritmo, bem como sua aplicação através de exemplos lúdicos, sem fazer o uso de computadores. As atividades propostas promovem as habilidades de escolha de uma representação apropriada para um problema e modelagem de aspectos relevantes de um problema tornando-o tratável, utilizando os conceitos de representação de dados, abstração, procedimentos, automatização e simulação, presentes no Pensamento Computacional.

A atividade foi dividida em sete tarefas, as quais foram propostas com uma abordagem lúdica, em níveis incrementais de formação e um processo de avaliação pré-definido, evidenciando seu potencial para a aprendizagem. O trabalho foi desenvolvido para ser adaptado a realidade da maioria das escolas públicas, afim de tornar a atividade de fácil aplicação em diversos contextos fora do ambiente acadêmico, fazendo uso de exercícios lúdicos, no qual assemelha-se a um jogo de tabuleiro.

Neste trabalho foram apresentadas metodologias e materiais a serem utilizados com a atividade proposta, adequando-se para introduzir o Pensamento Computacional em turmas do quinto ano do Ensino Fundamental de escolas públicas municipais. As metodologias apresentadas referem-se a cada tarefa proposta e os materiais utilizados foram pensados de forma a manter o baixo custo de obtenção e maior durabilidade.

A direção que toma a proposta deste trabalho indica a importância de iniciativas em desenvolver o Pensamento Computacional no ensino básico. Utilizando desta premissa, futuramente este trabalho proposto será aplicado em turmas do quinto ano do ensino fundamental da rede pública municipal de Pelotas.

\section{Referências}

Andrade, D., Carvalho, T., Silveira, J., Cavalheiro, S., Foss, L., Fleischmann, A., Aguiar, M., and Reiser, R. (2013). Proposta de atividades para o desenvolvimento do pensamento pomputacional no ensino fundamental. Workshop de Informática na Escola.

Bell, T., Witten, I. H., and Fellows, M. (2011). Computer Science Unplugged.

Bezerra, F. (2014). Bem mais que os bits da computação desplugada. In Anais do Workshop de Informática na Escola - WIE 2014, pages 116-125.

Costa, T., Batista, A., Maia, M., Almeida, L., and Farias, A. (2012). Trabalhando fundamentos de computação no nível fundamental: experiência de licenciandos em computação da universidade federal da paraíba. In XX Workshop sobre Educação em Computação, pages 1-4.

CSTA, ISTE, and NSF (2010). Computational thinking leadership toolkit. Disponível em http://csta.acm.org/Curriculum/sub/CurrFiles/. Acesso: Abril/2015. 
EXP-PC (2015). Explorando o pensamento computacional para a qualificação do ensino fundamental. http://wp.ufpel.edu.br/pensamentocomputacional/pt/. Acesso em Maio de 2015 .

Gonçalves, D., da Silva, G. M., da Luz, R. S., and Silva, E. P. (2013). Relato de experiência de alunos do curso de licenciatura em computação do ifmg-campus ouro branco na utilização de objetos de aprendizagem desplugados e do scratch como instrumentos no ensino de programação. Workshops do Congresso Brasileiro de Informática na Educação.

Linn, M., Aho, A. V., Blake, M. B., Constable, R., Kafai, Y. B., Kolodner, J. L., Snyder, L., Wilensky, U., Lin, H. S., Williams, E., and Bradley, S. (2010). Report of a Workshop on The Scope and Nature of Computational Thinking. National Academies Press.

Scaico, P. D., de Lima, A. A., da Silva, J. B. B., Azevedo, S., Paiva, L. F., Raposo, E. H. S., Alencar, Y., and Mendes, J. P. (2012). Programação no ensino médio: Uma abordagem de ensino orientado ao design com scratch. In Anais do Workshop de Informática na Escola - WIE 2012.

SEB, SECADI, SETEC, and CNE (2013). Diretrizes Curriculares Nacionais para Educação Básica. MEC.

SEF (1997). Parâmetros curriculares nacionais. MEC.

Silva, A., Moraes, D. A., and Batista, S. C. (2014a). Objetos de aprendizagem em scratch para estudo de saneamento básico: Percepções de alunos e professores. In Anais do Workshop de Informática na Escola - WIE 2014, pages 370-379.

Silva, T., Silva, A., and Melo, J. (2011). Ensino de algoritmos a nível médio utilizando música e robótica: Uma abordagem lúdica. In Anais do XIX Workshop sobre Educação em Computação - WEI 2011, pages 1-4.

Silva, T. R., Araujo, G. G., and da Silva Aranha, E. H. (2014b). Oficinas itinerantes de scratch e computação desplugada para professores como apoio ao ensino de computação - um relato de experiência. In Anais do Workshop de Informática na Escola - WIE 2014, pages 380-389.

Sousa, D. F. (2013). Desenvolvendo a lógica e algoritmos no ensino médio. In Anais do Workshop de Informática na Escola - WIE 2013, pages 101-109.

Torezani, C., da Costa Chagas, L. B., and de Lira Tavares, O. (2013). Newprog - um ambiente online para crianças aprenderem programação de computadores. In Anais do Workshop de Informática na Escola - WIE 2013, pages 140-149.

Viel, F., Raabe, A., and Zeferino, C. (2014). Introdução à programação e à implementação de processadores por estudantes do ensino médio. In Anais do Workshop de Informática na Escola - WIE 2014, pages 248-257.

Wing, J. M. (2006). Computational thinking. Communications of the ACM, 49(3):33-35.

Wing, J. M. (2011). Research notebook: Computational thinking-what and why? Disponível em http://www.cs.cmu.edu/link/research-notebook-computational-thinkingwhat-and-why. 